\section{Evaluación de presencia y severidad de periodontitis en pacientes chilenos con artritis reumatoide atendidos en el Hospital Padre Hurtado}

\author{
SEBASTIÁN IBÁÑEZ V. ${ }^{1,2}$, CRISTINA FERREIRO ${ }^{\mathrm{a}}$, ANDRÉS CONTRERAS $^{2, \mathrm{~b}}$, \\ OMAR VALENZUELA L. ${ }^{1,2}$, NICOLÁS GIADALAH ${ }^{\mathrm{a}}$, VICENTE JARA ${ }^{3}$, \\ FRANCISCA JAIME $^{4}$, ELENA JARPA ${ }^{1}$
}

\section{Frequency and severity of periodontitis among patients with rheumatoid arthritis}

\begin{abstract}
Background: Periodontitis may have a triggering and aggravating role of various medical conditions, including rheumatoid arthritis. Aim: To evaluate the periodontal status in Chilean patients with rheumatoid arthritis (RA), treated in a public hospital. Patients and Methods: A trained professional conducted a periodontal examination in 40 patients with RA aged 23 to 73 years (85\% women). When present, the severity of periodontitis and its relationship with gender, smoking, age, corticosteroids dose and AR activity were assessed. AR activity was evaluated using the Disease Activity Score Calculator for Rheumatoid Arthritis (DAS 28). Results: Thirty five of the 40 patients had periodontitis and in 13, it was severe. Men, smokers, and older patients had more severe stages. Patients using higher doses of corticosteroids had lower severity of periodontitis. No relationship between the severity of periodontitis and AR activity was found. Conclusions: Periodontitis is common and severe in patients with $R A$, and is influenced by gender, age, smoking and corticosteroid dose.
\end{abstract}

(Rev Med Chile 2015; 143: 1539-1545)

Key words: Arthritis, Rheumatoid; Glucocorticoids; Periodontitis.
${ }^{1}$ Reumatólogo, Hospital Padre Hurtado, Clínica Alemana de Santiago.

${ }^{2}$ Docente Facultad de Medicina Clínica Alemana-Universidad del Desarrollo.

${ }^{3}$ Residente Medicina Interna, Facultad de Medicina Clínica Alemana-Universidad del Desarrollo.

${ }^{4}$ Gastroenteróloga pediátrica, Pontificia Universidad Católica de Chile.

aEstudiante Odontología, Facultad de Medicina Clínica Alemana-Universidad del Desarrollo.

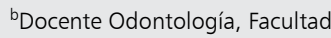
de Medicina Clínica AlemanaUniversidad del Desarrollo.

Recibido el 7 de julio de 2015, aceptado el 28 de septiembre de 2015 .

Correspondencia a: Sebastián Ibáñez Vodnizza Av. Manquehue 1410, piso 7 , Vitacura, Santiago, Chile. sibanez@alemana.cl
$\mathrm{L}$ a artritis reumatoide (AR) es una enfermedad autoinmue, sistémica, crónica y destructiva, caracterizada por acumulación y persistencia de infiltrados inflamatorios en la membrana sinovial, y por la inflamación de articulaciones, tendones y estructuras periarticulares ${ }^{1,2}$.

Sin tratamiento puede conducir a la destrucción de hueso y cartílago articular, de ligamentos y tejidos blandos, lo que provoca discapacidad grave e impacto clínicamente importante en la calidad de vida, con efectos sustanciales en términos de pérdida de productividad y costos ${ }^{3}$. En Chile la AR afecta aproximadamente al $0,46 \%$ de la población ${ }^{4}$. Las enfermedades periodontales son enfermedades que afectan a los tejidos que sostienen los dientes.
El primer paso es la gingivitis, una respuesta inmune inflamatoria a la colonización bacteriana de las superficies del diente, sin pérdida de hueso. La gingivitis puede progresar hacia periodontitis, una etapa avanzada y más grave de la enfermedad de las encías, que incluye la pérdida de hueso alveolar, llevando eventualmente a pérdida de dientes ${ }^{5}$. La periodontitis es una infección oral crónica causada por reacciones inflamatorias a bacterias gram-negativas anaeróbicas, y afecta a alrededor de 35 a $50 \%$ de los adultos en estudios internacionales, aunque las diferecnias en las prevalencias puede estar dada por distintas definiciones de caso $^{6,7}$. En Chile la prevalencia de periodontitis fue de $93,85 \%$ al considerar $3 \mathrm{~mm}$ de perdida de inserción y 
$38,65 \%$ al considerar $6 \mathrm{~mm}$ en personas entre los 35 y 44 años, y 97,58\% y 69,35\%, al considerar 3 y $6 \mathrm{~mm}$ respectivamente, entre los 65 y 76 años según un estudio publicado el $2010^{8}$, donde además identificaron como factores de riesgo para mayor severidad el ser hombre, fumador, de mayor edad $\mathrm{y}$ de menor nivel educacional.

El objetivo de este estudio transversal, descriptivo, es evaluar el estado periodontal de pacientes chilenos que sufren de $\mathrm{AR}$, atendidos en el Hospital Padre Hurtado, determinando la presencia y gravedad de periodontitis, y su posible relación con la actividad de la AR y otros datos demográficos.

\section{Pacientes y Método}

\section{Pacientes}

Se incluyeron en el estudio pacientes mayores de 18 años, con diagnóstico de AR según criterio ACR 2010, atendidos en forma consecutiva en el Hospital Padre Hurtado, hospital público perteneciente al Servicio de Salud Sur Oriente de Santiago. Se excluyeron a los que hubieran recibido tratamiento periodontal en los 6 meses previos, a los con menos de 12 piezas dentales naturales, pacientes con diabetes mellitus, a los que hubieran recibido terapia antibiótica en los últimos 3 meses, a las pacientes embarazadas, a los con diagnóstico de síndrome de Sjögren, y a los usuarios de más de $20 \mathrm{mg}$ de prednisona al día. En cada paciente se calculó el puntaje de actividad de AR DAS 28 (VHS) previo a la evaluación odontológica.

Se registraron las variables tabaquismo y dosis de corticoides.

\section{Evaluación periodontal}

Un periodoncista previamente entrenado, que desconocía la actividad de AR de los pacientes, realizó el examen periodontal, que consistió en el sondaje periodontal de las piezas dentarias naturales remanentes en boca, exceptuando los terceros molares. Sólo se consideraron aquellos terceros molares que se encontraban aislados, mesializados, siendo el único molar remanente por cada grupo. Se excluyeron piezas dentarias rotadas, con coronas sobrextendidas o sobrecontorneadas, coronas dentarias fracturadas y aquellas piezas dentarias con límite amelocementario (LAC) difícil de determinar. La mediciones se realizaron con la sonda periodontal calibrada Hu-Friedy de la Universidad Carolina del Norte número 15.

Se midieron los siguientes parámetros clínicos: 1) Número de piezas dentarias; 2) Profundidad al sondaje (PS); 3) Nivel de inserción clínica (NIC) y 4) Sangrado al sondaje (SS). Esta información fue registrada en un periodontograma. Las mediciones de PS y NIC fueron aproximadas al milímetro más cercano. Se midieron seis sitios en cada diente (por vestibular y palatino/lingual: mesial, vestibular y distal).

Para el diagnóstico de enfermedad periodontal, se consideró como profundidad de sondaje a la distancia medida desde el margen gingival libre hasta el fondo de saco. El nivel de inserción clínica se midió desde el LAC hasta el fondo de saco. La inflamación gingival se determinó por el sangrado al sondaje, expresada en porcentaje por los sitios que sangran.

Según la definición de caso de Eke, Page, Wei y colaboradores se catalogó como periodontitis leve la pérdida de inserción mayor o igual a $3 \mathrm{~mm}$ de 2 o más sitios y 2 o más sitios interproximales con una profundidad del sondaje mayor o igual a $4 \mathrm{~mm}$, que no corresponda al mismo diente o un sitio con una profundidad de sondaje mayor o igual $5 \mathrm{~mm}$. Periodontitis moderada se definió como la pérdida de inserción mayor o igual a $4 \mathrm{~mm}$ de 2 o más sitios o dos o más sitios interproximales con una profundidad al sondaje mayor o igual a $5 \mathrm{~mm}$ que no correspondan al mismo diente. Periodontitis severa se definió como la pérdida de inserción mayor o igual a $6 \mathrm{~mm}$ de 2 o más sitios que no corresponda al mismo diente y 1 o más sitios interproximales con una profundidad al sondaje mayor o igual a $5 \mathrm{~mm}^{9}$.

\section{Ética}

Todos los procedimientos respetaron las normas éticas concordantes con la Declaración de Helsinki, y fueron revisados y aprobados por el comité ético científico de la facultad de Medicina Clínica Alemana-Universidad del Desarrollo. Todos los participantes firmaron consentimiento informado.

\section{Análisis estadístico}

Los datos se analizaron utilizando el software SPSS v.20.0. Para evaluar diferencias entre las variables categóricas se utilizó test de chi-cuadrado, para evaluar diferencias entre variables categóri- 


\section{Tabla 1. Características de los participantes}

\begin{tabular}{|c|c|}
\hline $\begin{array}{l}\text { Características } \\
\text { sociodemográficas }\end{array}$ & $\begin{array}{c}\text { Total } \\
n=40\end{array}$ \\
\hline $\begin{array}{l}\text { Sexo } \\
\text { - Femenino } \\
\text { - Masculino }\end{array}$ & $\begin{array}{c}n \quad(\%) \\
34(85 \%) \\
6(15 \%)\end{array}$ \\
\hline Edad, mediana (rango) & 55,5 años (23-73) \\
\hline $\begin{array}{l}\text { Tabaco } \\
\text { - No fumador } \\
\text { - Fumador }\end{array}$ & $\begin{array}{c}n \quad(\%) \\
27(67,5 \%) \\
13(32,5 \%)\end{array}$ \\
\hline $\begin{array}{l}\text { Dosis de prednisona, mediana } \\
\text { (rango intercuartil) } \\
\text { - No consume } \\
\text { - } 5 \mathrm{mg} / \text { día } \\
\text { - } 7,5 \mathrm{mg} / \text { día } \\
\text { - } 10 \mathrm{mg} / \text { día } \\
\text { - } 15 \mathrm{mg} / \text { día } \\
\text { - } 17,5 \mathrm{mg} / \text { día } \\
\text { - } 20 \mathrm{mg} / \text { día }\end{array}$ & $\begin{array}{c}6,25 \mathrm{mg} / \mathrm{d}(5-10) \\
\mathrm{n} \quad(\%) \\
2 \quad(5 \%) \\
18(45 \%) \\
4(10 \%) \\
12(30 \%) \\
2 \quad(5 \%) \\
1 \quad(2,5 \%) \\
1 \quad(2,5 \%)\end{array}$ \\
\hline $\begin{array}{l}\text { Actividad de AR, mediana } \\
\text { (rango intercuartil) } \\
\text { - Remisión: DAS } 28<2,6 \\
\text { - Leve: } 2,6 \leq \text { DAS } 28 \leq 3,2 \\
\text { - Moderada: } 3,2<\text { DAS } 28 \leq 5,1 \\
\text { - Severa: DAS } 28>5,1\end{array}$ & $\begin{array}{c}3,945(3,03-4,92) \\
n \quad(\%) \\
7(17,5 \%) \\
4(10 \%) \\
23(57,5 \%) \\
6(15 \%)\end{array}$ \\
\hline $\begin{array}{l}\text { Severidad de Periodontitis } \\
\text { - Sano } \\
\text { - Leve } \\
\text { - Moderada } \\
\text { - Severa }\end{array}$ & $\begin{array}{c}n \quad(\%) \\
5(12,5 \%) \\
1 \quad(2,5 \%) \\
21(52,5 \%) \\
13(32,5 \%)\end{array}$ \\
\hline $\begin{array}{l}\text { SS } \% \text { mediana, (rango intercuartil) } \\
-\leq 20 \\
->20\end{array}$ & $\begin{array}{l}25(15-42) \\
n \quad(\%) \\
13(32,5 \%) \\
27(67,5 \%)\end{array}$ \\
\hline
\end{tabular}

cas y numéricas se utilizó U de Mann Whitney. Se consideró estadísticamente significativo un $\mathrm{p}<0,05$.

\section{Resultados}

\section{Características de los participantes}

Cincuenta y dos pacientes cumplieron los criterios para ingresar al estudio, de los cuales 12 no asistieron a la evaluación odontológica por motivos personales. Se describen las características de los 40 pacientes en la Tabla 1. De los 40 pacientes, 35 sufrían de periodontitis (87,5\%), en 13 casos severa $(32,5 \%)$.

\section{Severidad de periodontitis}

Los hombres presentaron estados más severos de periodontitis $(\mathrm{p}=0,038)$ (Tabla 2$)$. Igualmente, aquellos pacientes de mayor edad $(\mathrm{p}=0,021)$ (Tabla 3), y los fumadores $(\mathrm{p}=0,011)$ (Tabla 4$)$ presentaron mayor severidad de periodontitis. En cuanto al uso de corticoides destaca que los pacientes que recibían mayores dosis presentaron menor severidad de periodontitis $(\mathrm{p}=0,012)(\mathrm{Ta}-$ bla 5). No se encontró diferencias significativas en cuanto a sexo y tabaquismo y su relación con la actividad de AR medida por DAS 28.

\section{Relación entre AR y periodontitis}

No se encontró relación estadísticamente significativa entre severidad de periodontitis y actividad de AR medida por DAS 28 ( $\mathrm{p}=0,6138$ ) (Tabla 6).

\section{Discusión}

Presentamos el primer reporte del estado periodontal de pacientes chilenos que sufren de AR.

Varios estudios recientes han demostrado

Tabla 2. Relación de género y severidad de periodontitis y actividad de AR

\begin{tabular}{|c|c|c|c|c|c|c|c|c|c|c|}
\hline \multirow[t]{2}{*}{ Sexo } & \multicolumn{4}{|c|}{ Severidad de periodontitis* } & \multirow[t]{2}{*}{ Total } & \multicolumn{4}{|c|}{ Actividad de artritis reumatoide } & \multirow[t]{2}{*}{ Tota } \\
\hline & Sano & Leve & Moderada & Severo & & Remisión & Leve & Moderada & Severa & \\
\hline Femenino & 5 & 1 & 20 & 8 & 34 & 6 & 3 & 20 & 5 & 34 \\
\hline Masculino & 0 & 0 & 1 & 5 & 6 & 1 & 1 & 3 & 1 & 6 \\
\hline Total & 5 & 1 & 21 & 13 & 40 & 7 & 4 & 23 & 6 & 40 \\
\hline
\end{tabular}

Los hombres presentaron estados más severos de periodontitis ( $p=0,038$; al comparar severos vs no severos $p<0,01)$. Sin diferencias en cuanto a actividad de AR. 
Tabla 3. Relación entre severidad de periodontitis y edad de los pacientes

\begin{tabular}{|cccc|}
\hline Severidad de periodontitis & Mediana edad & Rango edad & Frecuencia \\
\hline Sano & 37 & $23-43$ & 5 \\
\hline Leve & 52 & $27-73$ & 1 \\
\hline Moderada & 48 & $32-68$ & 21 \\
Severa & 54 & $23-73$ & 40 \\
\hline Total & 50,5 & 52 & 43 \\
\hline
\end{tabular}

Los pacientes de mayor edad presentaron estados más severos de periodontitis $(p=0,021)$.

Tabla 4. Relación de tabaquismo y severidad de periodontitis y actividad de AR

\begin{tabular}{|c|c|c|c|c|c|c|c|c|c|c|}
\hline \multirow{2}{*}{$\begin{array}{l}\text { Consumo } \\
\text { de tabaco }\end{array}$} & \multicolumn{4}{|c|}{ Severidad de periodontitis } & \multirow[t]{2}{*}{ Total } & \multicolumn{4}{|c|}{ Actividad de artritis reumatoide } & \multirow[t]{2}{*}{ Total } \\
\hline & Sano & Leve & Moderada & Severa & & Remisión & Leve & Moderada & Severa & \\
\hline No fumador & 5 & 0 & 17 & 5 & 27 & 7 & 3 & 14 & 3 & 27 \\
\hline Fumador & 0 & 1 & 4 & 8 & 13 & 0 & 1 & 9 & 3 & 13 \\
\hline Total & 5 & 1 & 21 & 13 & 40 & 7 & 4 & 23 & 6 & 40 \\
\hline
\end{tabular}

Los fumadores presentaron estados más severos de periodontitis $(p=0,011)$. Sin diferencias en actividad de artritis reumatoide.

Tabla 5. Relación entre dosis de prednisona y severidad de periodontitis

\begin{tabular}{|lccccc|}
\hline $\begin{array}{l}\text { Dosis de prednisona } \\
\text { mg/día }\end{array}$ & \multicolumn{4}{c}{ Severidad de periodontitis } \\
& Sano & Leve & Moderado & Severo & Total \\
\hline$\leq 5 \mathrm{mg}$ & 2 & 0 & 11 & 7 & 20 \\
\hline $7,5 \mathrm{mg}$ y $10 \mathrm{mg}$ & 1 & 0 & 9 & 6 & 16 \\
\hline 15 a $20 \mathrm{mg}$ & 2 & 1 & 21 & 0 & 4 \\
\hline Total & 5 & 1 & 13 & 40 \\
\hline
\end{tabular}

Los pacientes con mayor dosis de corticoides presentaron periodontitis menos severa $(p=0,012)$.

Tabla 6. Relación entre severiad de periodontits y actividad de AR

\begin{tabular}{|c|c|c|c|c|c|}
\hline \multirow{2}{*}{$\begin{array}{l}\text { Severidad de } \\
\text { periodontitis }\end{array}$} & \multicolumn{5}{|c|}{ Actividad de la artritis reumatoidea } \\
\hline & Remisión & Leve & Moderada & Severa & Total \\
\hline Sano & 1 & 0 & 3 & 1 & 5 \\
\hline Leve & 0 & 0 & 0 & 1 & 1 \\
\hline Moderada & 4 & 2 & 12 & 3 & 21 \\
\hline Severa & 2 & 2 & 8 & 1 & 13 \\
\hline Total & 7 & 4 & 23 & 6 & 40 \\
\hline
\end{tabular}

No se encontró relación estadísticamente significativa entre la severidad de periodontitis y la actividad de AR. 
que la periodontitis podría tener una influencia importante en la carga inflamatoria sistémica, y podría desencadenar o empeorar muchas condiciones médicas, incluyendo infarto de miocardio y accidente cerebrovascular ${ }^{10,11}$, el control glicémico en pacientes con diabetes ${ }^{12,13}$, los nacimientos prematuros ${ }^{14,15}$, ocurrencia de enfermedad pulmonar obstructiva crónica y complicaciones respiratorias ${ }^{16}$, e incluso disfunción eréctil ${ }^{17}$. AR y periodontitis comparten algunas características: ambas son enfermedades inflamatorias crónicas con influencia genética y ambiental, y desequilibrio inmunorregulador, y ambas conducen a la destrucción de tejidos conectivos y duros ${ }^{18,19}$. Un estudio reportó que la frecuencia de AR es significativamente mayor en pacientes con enfermedad periodontal que en los sujetos sin periodontitis $(3,95 \% \text { frente a } 0,66 \%)^{20}$.

Otros estudios han reportado una mayor incidencia de falta de dientes, placa dental, una mayor profundidad de la bolsa periodontal, o peores niveles de inserción clínica en los pacientes con $\mathrm{AR}^{19-21}$. La prevalencia reportada de periodontitis en $\mathrm{AR}$ es variable en estudios internacionales, entre 25 y 97\%, lo que depende en parte de las distintas definiciones de caso $^{22-25}$. Es probable que los pacientes con AR tengan más dificultades para lograr una buena salud oral debido al dolor en las articulaciones o a la limitación funcional ${ }^{21,26}$, pero existe la hipótesis de que la periodontitis podría ser un factor de riesgo o un factor agravante para la AR. En particular, el papel de Porphyromonas gingivalis, una bacteria periodontopatógena bien conocida, ha sido ampliamente estudiado, porque esta bacteria oral posee una enzima endógena, peptidil arginina deaminasa ${ }^{27}$, capaz de citrulinar residuos de arginina, uno de los primeros pasos en el desarrollo de la $\mathrm{AR}^{28,30}$.

Nuestros resultados son concordantes en cuanto a prevalencia y factores de riesgo de severidad de periodontitis (ser hombre, mayor edad, tabaquismo) con los obtenidos en el principal estudio realizado en población general chilena ${ }^{8}$, donde evaluaron a 1.561 pacientes. Agregamos a estos datos la menor severidad de periodontitis en pacientes que utilizan mayor dosis de corticoides.

Varios investigadores han postulado que la terapia corticoidal tendría un efecto terapeútico en la enfermedad periodontal. Pero la evidencia no es concluyente, se ha reportado aumento de osteoporosis mandibular y candidiasis oral, pero no mejoría ni deterioro de periodontitis ${ }^{31-34}$. En nuestro estudio se estableció como criterio de exclusión el uso de más de $20 \mathrm{mg}$ de prednisona al día, pero ningún paciente fue excluido por este criterio.

Nuestro estudio tiene algunas limitaciones, el número de pacientes evaluados, los pocos hombres evaluados, nos impiden concluir con mayor certeza sobre los factores de riesgo para sufrir de periodontitis más o menos severa.

Un punto importante que sigue siendo una incognita es el rol del estado periodontal en la actividad de la AR. Algunos estudios han demostrado que el tratamiento periodontal puede inducir una disminución significativa de la velocidad de sedimentación globular (VHS) o de la actividad de la enfermedad (DAS28) ${ }^{35-38}$. Pero estos trabajos son pequeños y de baja calidad, por lo que se requieren otros estudios, especialmente ensayos controlados aleatorizados, para determinar si el tratamiento de la periodontitis en los pacientes con AR resulta en la mejora de resultados clínicos o de calidad de vida. Los datos obtenidos en este estudio sirvieron como base para el diseño de un estudio prospectivo, randomizado, controlado, actualmente en curso en nuestro centro, cuyo objetivo es evaluar el efecto del tratamiento periodontal en la actividad de la AR.

\section{Referencias}

1. Mercado FB, Marshall RI, Bartold PM. Inter-relationships between rheumatoid arthritis and periodontal disease. A review. J Clin Periodontol 2003; 30: 761-72.

2. Lee DM, Weinblatt ME. Rheumatoid arthritis. Lancet 2001; 358: 903-11.

3. Kvien TK, Uhlig T. Quality of life in rheumatoid arthritis. Scand J Rheumatol 2005; 34: 333-41.

4. Riedemann JP, Maluje V. Epidemiología de las Enfermedades Reumatológicas en Chile. Informe Proyecto Fondecyt 1930390, 1993.

5. ADA. International workshop for a classification of periodontal diseases and conditions papers. Ann Periodontol 1999; 4: 1-112.

6. Bourgeois D, Bouchard P, Mattout C. Epidemiology of periodontal status in dentate adults in France, 20022003. J Periodontal Res 2007; 42: 219-27.

7. Albandar JM, Brunelle JA, Kingman A. Destructive periodontal disease in adults 30 years of age and older in the United States, 1988-1994. J Periodontol 1999; 70: 13-29. 
8. Gamonal J, Mendoza C, Espinoza I, Muñoz A, Urzúa I, Aranda W, et al. Clinical attachment loss in Chilean adult population: First Chilean National Dental Examination Survey. J Periodontol 2010; 81 (10): 1403-10. doi: 10.1902/jop.2010.100148.

9. Eke PI, Page RC, Wei L, Thornton-Evans G, Genco RJ. Update of the case definitions for population-based surveillance of periodontitis. J Periodontol 2012; 83 (12): 1449-54. doi: 10.1902/jop.2012.110664.

10. Janket SJ, Baird AE, Chuang SK, Jones JA. Meta-analysis of periodontal disease and risk of coronary heart disease and stroke. Oral Surg Oral Med Oral Pathol Oral Radiol Endod 2003; 95: 559-69.

11. Blaizot A, Vergnes J, Nuwwareh S, Amar J, Sixou M. Periodontal diseases and cardiovascular events: meta-analysis of observational studies. Int Dent J 2009; 59: 197-209.

12. Vergnes JN, Arrivé E, Gourdy P, Hanaire H, Rigalleau $\mathrm{V}$, Gin $\mathrm{H}$, et al. Periodontal treatment to improve glycaemic control in diabetic patients: study protocol of the randomized, controlled DIAPERIO trial. Trials 2009; 10: 65.

13. Darré L, Vergnes JN, Gourdy P, Sixou M. Efficacy of periodontal treatment on glycaemic control in diabetic patients: A meta-analysis of interventional studies. Diabetes Metab 2008; 34: 497-506.

14. Nabet C, Lelong N, Colombier ML, Sixou M, Musset $\mathrm{AM}$, Goffinet F, et al. Maternal periodontitis and the causes of preterm birth: the case-control Epipap study. J Clin Periodontol 2010; 37: 37-45.

15. Vergnes JN, Sixou M. Preterm low birth weight and maternal periodontal status: a meta- analysis. Am J Obstet Gynecol 2007; 196: 135-7.

16. Azarpazhooh A, Leake JL. Systematic review of the association between respiratory dieases and oral health. J Periodontol 2006; 77: 1465-82.

17. Zadik Y, Bechor R, Galor S, Justo D, Heruti RJ. Erectile dysfunction might be associated with chronic periodontal disease: two ends of the cardiovascular spectrum. J Sex Med 2009; 6: 1111-6.

18. Mercado F, Marshall RI, Klestov AC, Bartold PM. Is there a relationship between rheumatoid arthritis and periodontal disease? J Clin Periodontol 2000; 27: 267-72.

19. Mercado FB, Marshall RI, Klestov AC, Bartold PM. Relationship between rheumatoid arthritis and periodontitis. J Periodontol 2001; 72: 779-87.

20. Pischon N, Pischon T, Kröger J, Gülmez E, Kleber BM, Bernimoulin JP, et al. Association among rheumatoid arthritis, oral hygiene, and periodontitis. J Periodontol 2008; 79: 979-86.

21. Ishi Ede P, Bertolo MB, Rossa C Jr, Kirkwood KL,
Onofre MA. Periodontal condition in patients with rheumatoid arthritis. Braz Oral Res 2008; 22: 72-7.

22. Ranade SB, Doiphode S. Is there a relationship between periodontitis and rheumatoid arthritis? J Indian Soc Periodontol 2012; 16 (1): 22-7. doi: 10.4103/0972124X.94599.

23. Scher JU, Ubeda C, Equinda M, Khanin R, Buischi Y, Viale A, et al. Periodontal disease and the oral microbiota in new-onset rheumatoid arthritis. Arthritis Rheum 2012; 64 (10): 3083-94. doi: 10.1002/art.34539.

24. Mikuls TR, Payne JB, Yu F, Thiele GM, Reynolds RJ, Cannon GW, et al. Periodontitis and Porphyromonas gingivalis in patients with rheumatoid arthritis. Arthritis Rheumatol 2014; 66 (5): 1090-100. doi: 10.1002/ art.38348.

25. de Smit M, Westra J, Vissink A, Doornbos-van der Meer B, Brouwer E, van Winkelhoff AJ. Periodontitis in established rheumatoid arthritis patients: a cross-sectional clinical, microbiological and serological study. Arthritis Res Ther 2012; 14 (5): R222. doi: 10.1186/ar4061.

26. Bessa-Nogueira RV, Vasconcelos BC, Duarte AP, Goes PS, Bezerra TP. Targeted assessment of the temporomandibular joint in patients with rheumatoid arthritis. J Oral Maxillofac Surg 2008; 66: 1804-11.

27. McGraw WT, Potempa J, Farley D, Travis J. Purification, characterization, and sequence analysis of a potential virulence factor from Porphyromonas gingivalis, peptidylarginine deiminase. Infect Immun 1999; 67: 3248-56.

28. Routsias JG, Goules JD, Goules A, Charalampakis G, Pikazis D. Autopathogenic correlation of periodontitis and rheumatoid arthritis. Rheumatology 2011; 50: 1189-93.

29. Bartold PM, Marshall RI, Haynes DR. Periodontitis and rheumatoid arthritis: a review. J Periodontol 2005; 76: 2066-74.

30. Rosenstein ED, Greenwald RA, Kushner LJ, Weissmann G. Hypothesis: the humoral immune response to oral bacteria provides a stimulus for the development of rheumatoid arthritis. Inflammation 2004; 28: 311-8.

31. Beeraka SS, Natarajan K, Patil R, Manne RK, Prathi VS, Kolaparthi VS. Clinical and radiological assessment of effects of long-term corticosteroid therapy on oral health. Dent Res J (Isfahan) 2013; 10 (5): 666-73.

32. Safkan B, Knuuttila M. Corticosteroid therapy and periodontal disease. J Clin Periodontol 1984; 11 (8): 515-22. doi:10.1111/j.1600-051X.1984.tb00903.x.

33. Heasman PA, Hughes FJ. Drugs, medications and periodontal disease. Br Dent J 2014; 217 (8): 411-9.

34. Scarabelot VL, Cavagni J, Medeiros LF, Detânico B, Rozisky JR, de Souza A, et al. Periodontal disease and high doses of inhaled corticosteroids alter NTPDase 
activity in the blood serum of rats. Arch Oral Biol 2014; 59 (8): 841-7. doi: 10.1016/j.archoralbio.2014.05.004.

35. Ribeiro J, Leão A, Novaes AB. Periodontal infection as a possible severity factor for rheumatoid arthritis. J Clin Periodontol 2005; 32: 412-6.

36. Ortiz P, Bissada NF, Palomo L, Han YW, Al-Zahrani MS, Panneerselvam A, Askari A. Periodontal therapy reduces the severity of active rheumatoid arthritis in patients treated with or without tumor necrosis factor inhibitors. J Periodontol 2009; 80: 535-40.

37. Al-Katma MK, Bissada NF, Bordeaux JM, Sue J, Askari $\mathrm{AD}$. Control of periodontal infection reduces the severity of active rheumatoid arthritis. J Clin Rheumatol 2007; 13: $134-7$.

38. Pinho M de N, Oliveira RD, Novaes AB Jr, Voltarelli JC. Relationship between periodontitis and rheumatoid arthritis and the effect of non-surgical periodontal treatment. Braz Dent J 2009; 20: 355-64. 